\title{
On the Density of the Ward Ansätze in the Space of Anti-Self-Dual Yang Mills Solutions
}

\author{
J. S. Ivancovich ${ }^{1}$, L. J. Mason ${ }^{2}$ and E. T. Newman ${ }^{1}$ \\ ${ }^{1}$ Department of Physics and Astronomy, University of Pittsburgh, Pittsburgh, PA 15260, USA \\ 2 New College, Oxford University, Oxford OX1 3BN, UK
}

\begin{abstract}
A general patching matrix $P$ for the twistor construction of antiself-dual Yang-Mills fields is approximated by a terminating Laurent series. The approximate patching matrix $P^{(m)}$ is triangularized (so that it becomes one of the Ward ansätze) and the associated Riemann-Hilbert problem is solved, thereby generating an anti-self-dual solution of the Yang-Mills equations. The approximate patching matrices and the associated (exact) anti-self-dual Yang-Mills solutions are then shown to converge on $P$ and its corresponding solution so that the Ward ansätze forms a dense subset in the solution space in the Weierstrass sense.
\end{abstract}

\section{Introduction}

For some time now there has been great interest in anti-self-dual (or self-dual) solutions of the Yang-Mills equations [1-6]. One of the reasons for this interest is that many of the familiar non-linear equations of mathematical physics (for example, the Bogomolny equation, the sine- and sinh-Gordan equations, the Toda-lattice equation, the stationary axial symmetric Einstein and EinsteinMaxwell equations, the KdV equation, the non-linear Schrödinger equation, and others) are symmetry reductions of the anti-self-dual Yang-Mills (ASDYM) equations for various gauge groups [7-10]. The ASDYM equations fall into the class of differential equations which are usually described as integrable or solvable [8]. Many solution generating techniques have been developed for the ASDYM equations, including the matrix-valued Sparling equation, the use of Lax pairs, the application of Bäcklund transformations to known seed solutions, and of most importance to this work, the twistor construction where twistor data is freely prescribed and the solution of a classical Riemann-Hilbert $(\mathrm{RH})$ problem is required in order to obtain the anti-self-dual field [11-13]. While various correspondences between these different methods have been known for some time, recently it has been shown that most can be directly obtained from a single construction [14].

Even though the ASDYM equations are in some technical sense solvable, obtaining explicit solutions given arbitrary data is difficult at best. It has been 
shown, for either the twistor construction $[3,5,11]$ or the Sparling construction [13], that if the appropriate matrix-valued data is of upper or lower triangular form then the general solution (in terms of quadratures) can be given. While the solutions obtained from this triangular data do not constitute the complete solution space of the ASDYM equations, it has been conjectured (at least for gauge groups $S U(2)$ or $S L(2, \mathbb{C})$ ) that they do form a dense subset in the solution space [5].

In this work, it is shown how arbitrary matrix-valued data for the twistor construction can be approximated by data which can then be triangularized; the explicit solution for this approximate data can then be given by quadratures. This exact solution from the approximate data, as this data approaches the exact data, converges to the solution of the exact data. The set of approximate (or triangularizable) data thus forms a dense subset (in the Weierstrass sense) in the entire data space and the solutions of this triangular data forms a dense subset in the solution space.

In Sect. II, a brief description of the ASDYM field equations and their correspondence with the twistor construction (and the RH problem) is given. Several of the important features of the twistor construction, e.g. certain types of gauge freedom, which will be exploited in the approximation scheme, are described. Section III contains an explicit method for obtaining the solutions of the ADYM equations via the RH splitting (twistor construction) for triangular data (for an arbitrary gauge group). (This is a direct extension of previous methods of solving the RH problem for two dimensional triangular data $[5,11]$. In Sect. IV, the procedure for approximating arbitrary $S L(2, \mathbb{C})$ data which can then be explicitly triangularized is described; the solution to this triangularized data can then be obtained by employing the method given in Sect. III. In Sect. V, the solutions to the approximate data are shown to converge to the solutions of the exact data as the approximate data converges to the exact data. The procedure described in Sect. IV is generalized to arbitrary $S L(n, \mathbb{C})$ data in Sect. VI. In conclusion, the importance of demonstrating the density of the solvable solution space for the ASDYM equations is discussed. Also, the prospect of obtaining similar results for the remaining solution generating techniques such as the Sparling equation are considered.

\section{Twistor Construction of the Anti-Self-Dual Yang-Mills Equations}

In this section, we review the ASDYM equations and describe our notation. The twistor construction for generating solutions is briefly introduced and the properties (gauge freedoms) of this construction are described. These gauge freedoms are extensively used in the approximation and triangularization scheme of Sects. IV and VI.

On Minkowski space $\mathbb{M}$ or an open set of $\mathbb{M}$, consider a trivial $n$-dimensional complex vector bundle $B=\mathbb{M} \times \mathbb{C}^{n}$ associated with some gauge group, $S L(n, \mathbb{C})$. We introduce the matrix-valued connection (or vector potential) $\gamma_{a}$, where a is the space-time index and the associated (Yang-Mills) gauge field $F$ defined by

$$
F_{a b}=\gamma_{b, a}-\gamma_{a, b}-\left[\gamma_{a}, \gamma_{b}\right] .
$$


The ASDYM equations are three matrix-valued differential equations on the connection,

$$
F_{a b}^{*}=-i F_{a b},
$$

where * is the Hodge duality operator given by

$$
F_{a b}^{*}=\frac{1}{2} \varepsilon_{a b c d} F^{c d}
$$

where $\varepsilon_{a b c d}$ is the alternating symbol and $\varepsilon_{0123}=-1$.

The ASDYM equations can be represented as the integrability conditions for a linear system as follows. Firstly they can be rewritten as [14]

$$
F_{a b} L^{a b}=F_{a b} \bar{M}^{a b}=F_{a b} N^{a b}=0
$$

where $L^{a b}, \bar{M}^{a b}$, and $N^{a b}$ are any three linearly independent self-dual antisymmetric tensors. These can be re-expressed as

$$
F_{a b} \bar{m}^{a b}=0,
$$

with $\bar{m}^{a b}$ a self-dual skew tensor given by

$$
\bar{m}^{a b}=L^{a b}+\bar{M}^{a b} \zeta+N^{a b} \zeta^{2},
$$

where $\zeta$ is an arbitrary point on the complex Riemann sphere, $\mathbb{C} \cup\{\infty\}, \mathbb{C P}^{1}$. A constant normalized null tetrad on $\mathbb{M}$ is introduced as $1^{a}, m^{a}, \bar{m}^{a}$, and $n^{a}$ with $1^{a} n_{a}=-m^{a} \bar{m}_{a}=1$, all other products vanishing. Define

and

$$
L^{a}(\zeta)=1^{a}+\zeta m^{a}
$$

$$
\bar{M}^{a}(\zeta)=\bar{m}^{a}+\zeta n^{a}
$$

$\bar{m}^{a b}$ can now be chosen as

$$
\begin{aligned}
\bar{m}^{a b} & =L^{[a}(\zeta) \bar{M}^{b]}(\zeta) \\
& =1^{[a} \bar{m}^{b]}+\zeta\left\{1^{[a} n^{b]}+m^{[a} \bar{m}^{b]}\right\}+\zeta^{2} m^{[a} n^{b]} .
\end{aligned}
$$

Applying (2.8) to (2.5), the ASDYM equations become

$$
F_{a b} L^{[a}(\zeta) \bar{M}^{b]}(\zeta)=0,
$$

which are the integrability conditions for the existence of solutions to

$$
L^{a}(\zeta)\left(\partial_{a}-\gamma_{a}\right) G=0, \quad \bar{M}^{a}(\zeta)\left(\partial_{a}-\gamma_{a}\right) G=0,
$$

with some matrix $G=G(x, \zeta)$. The skew tensor $\bar{m}^{a b}$ at any point in $\mathbb{M}$ defines a family of self-dual null two-surfaces through that point. For each value of $\zeta, \bar{m}^{a b}$ defines a self-dual null two-plane through the point, the vectors $L^{a}(\zeta)$ and $\bar{M}^{a}(\zeta)$ being two linearly independent vectors within the plane. The set of all such two surfaces in complexified Minkowski space $(\mathbb{C M})$ is projective dual twistor space $\mathbb{P T} \equiv \mathbb{C P}^{3}$. It can be coordinated by the local coordinates

$$
L=L_{a}(\zeta) x^{a}, \bar{M}=\bar{M}_{a}(\zeta) x^{a}, \text { and } \zeta .
$$

Note that $L, \bar{M}$, and $\zeta$ are complex and $x^{a}$ are coordinates on $\mathbb{C M}$; $L$ and $\bar{M}$ satisfy

$$
L^{a}(\zeta) \partial_{a} L=L^{a}(\zeta) \partial_{a} \bar{M}=0 \quad \text { and } \quad \bar{M}^{a}(\zeta) \partial_{a} L=\bar{M}^{a}(\zeta) \partial_{a} \bar{M}=0
$$


A correspondence exists between ASDYM fields and holomorphic vector bundles over a region of $\mathbb{P T}[1,12]$. Holomorphic vector bundles on $\mathbb{P} T$ can be described as follows; Consider $\mathbb{P T}$ covered by two patches $U_{0}$ and $U_{\infty}$ such that

$$
U_{0}=\{\mathbb{P T} / \zeta=\infty\} \quad \text { and } U_{\infty}=\{\mathbb{P T} / \zeta=0\},
$$

and $U_{0} \cap U_{\infty}$ projects to an annular region in the $\zeta$-Riemann sphere containing the equator, the circle $|\zeta|=1$. A holomorphic vector bundle can be specified by an $n \times n$ matrix of functions $P(L, \bar{M}, \zeta)$ holomorphic in the annular region $U_{0} \cap U_{\infty}$, known as the patching or transition matrix; it transforms a frame for the bundle, defined on $U_{0}$ to one defined on $U_{\infty}$. Specifying the patching matrix $P$ determines the bundle which in turn determines an anti-self-dual Yang-Mills connection and field (associated with the indicated vector bundle); the problem then is to explicitly obtain the connection and fields on $\mathbb{M}$ from the patching matrix $P$. These fields, on $\mathbb{M}$, can be obtained by "splitting" the patching matrix, i.e. by writing it (using (2.10) to express $L$ and $\bar{M}$ as functions of $x^{a}$ and $\zeta$ ) as

$$
P(L, \bar{M}, \zeta)=G_{\infty}\left(x^{a}, \zeta\right)^{-1} G_{0}\left(x^{a}, \zeta\right)
$$

or

$$
G_{\infty}\left(x^{a}, \zeta\right) P(L, \bar{M}, \zeta)=G_{0}\left(x^{a}, \zeta\right),
$$

where $G_{0}\left(x^{a}, \zeta\right)$ and $G_{\infty}\left(x^{a}, \zeta\right)$ are $n \times n$ matrices of functions, holomorphic on $\hat{U}_{0}=\left\{\mathbb{M} \times \mathbb{C P}^{1} / \zeta=\infty\right\}$ and $\hat{U}_{\infty}=\left\{\mathbb{M} \times \mathbb{C P}^{1} / \zeta=0\right\}$ respectively. This splitting is the solution of a classical matrix-valued Riemann-Hilbert $(\mathrm{RH})$ problem for each value of $x^{a}$ (the space-time points acting as parameters in the splitting).

Remark 1. The general splitting of any non-singular holomorphic matrix of functions is given by

$$
P(\zeta)=G_{\infty}^{-1}(\zeta) H(\zeta) G_{0}(\zeta)
$$

where $G_{0}(\zeta)$ and $G_{\infty}(\zeta)$ are the matrices given above and $H(\zeta)$ is a diagonal matrix whose entries are integral powers of $\zeta$. This splitting is known as a Birkhoff decomposition. If $H(\zeta)=1$ the above splitting is known as a Riemann-Hilbert (RH) problem. A generic patching matrix $P(\zeta)$ will possess a Birkhoff decomposition with $H(\zeta)=1$ and therefore possess a RH splitting [15].

In the scalar case the RH problem is to find $\Phi_{\infty}(\zeta)$ and $\Phi_{0}(\zeta)$ such that

$$
F(\zeta)=\Phi_{\infty}^{-1}(\zeta) \Phi_{0}(\zeta)
$$

where $\Phi_{\infty}(\zeta)$ is a holomorphic function on $U_{\infty}, \Phi_{0}(\zeta)$ is a holomorphic function on $U_{0}$, and $F(\zeta)$ is a given holomorphic function on the overlap $U_{0} \cap U_{\infty}$. The solution to this splitting is obtained by taking logarithms of both sides

$$
\ln F(\zeta)=\ln \Phi_{0}(\zeta)-\ln \Phi_{\infty}(\zeta)
$$

and applying the Cauchy integral theorem, provided that the function $\ln F(\zeta)$ is single-valued around any contour linking the annulus (if $\ln F(\zeta)$ is not single-valued, one must first multiply $F(\zeta)$ by $\zeta^{m}$ for some integral $m$ before taking logs in order to ensure that the logarithm is single valued).

Associated with the scalar $\mathrm{RH}$ problem is the non-homogeneous $\mathrm{RH}$ (or Hilbert) 
problem given by the splitting

$$
F(\zeta)=\hat{\Phi}_{\infty}^{-1}(\zeta) \hat{\Phi}_{0}(\zeta)+\hat{\Phi}_{\infty}^{-1}(\zeta) K(\zeta),
$$

where $K(\zeta)$ is a given holomorphic function on the overlap $U_{0} \cap U_{\infty}$. Solutions to the non-homogeneous problem are obtained by reduction to an additive $\mathrm{RH}$ problem using the solution to the homogeneous problem [16]. When the $\zeta^{m}$ factor is nontrivial for the associated homogeneous problem, there are obstructions to solving the nonhomogeneous problem. These obstructions arise because the problem reduces to the following additive $\mathrm{RH}$ problem:

$$
K^{\prime}(\zeta)=\zeta^{m} g_{0}(\zeta)-g_{\infty}(\zeta)
$$

One can only solve this RH problem for $g_{0}$ and $g_{\infty}$ when the Laurent expansion for $K^{\prime}$ has no powers of $\zeta$ between 1 and $m-1$ since $g_{\infty}(\zeta)$ only contains powers of $\zeta$ smaller than 1 , and $\zeta^{m} g_{0}(\zeta)$ only contains powers greater than $m-1$. These obstructions play an essential and subtle role in the solution of the Ward ansatze.

Given $P$, the solution to the RH problem is unique up to premultiplication by a matrix depending only on $x^{a}$. Given $G_{0}$ or $G_{o \infty}$, the ASDYM connection $\gamma_{a}$ is defined by

$$
\gamma_{a} L^{a}(\zeta)=L^{a}(\zeta) G_{0, a} G_{0}^{-1}=L^{a}(\zeta) G_{\infty, a} G_{\infty}^{-1}
$$

and

$$
\gamma_{a} \bar{M}^{a}(\zeta)=\bar{M}^{a}(\zeta) G_{0, a} G_{0}^{-1}=\bar{M}^{a}(\zeta) G_{\infty, a} G_{\infty}^{-1} .
$$

These expressions given the four components of the connection $\gamma_{a}\left(x^{a}\right)$ which are automatically solutions of the ASDYM equations, (2.9) [14]. This can be seen from the fact that (2.13) implies that $G_{0}$ and $G_{\infty}$ solve the linear system (2.9b) so that the integrability conditions (2.9a) are automatically solved.

Remark 2. The first equality in (2.13a) shows that $\gamma_{a} L^{a}(\zeta)$ is regular for $|\zeta|<$ infinity and the second equality shows that it has a simple pole at infinity arising from that in $L^{a}(\zeta)$ (cf. (2.7)). Therefore $\gamma_{a} L^{a}(\zeta)$ is linear in $\zeta$ by an elementary extension of Liouville's theorem. By applying a similar argument to (2.13b) it can be seen that $(2.13 \mathrm{a}, \mathrm{b})$ do indeed determine just the four components of the connection.

The patching matrix $P$ is effectively a freely specified holomorphic $S L(n, \mathbb{C})$ function of three variables for $S L(n, \mathbb{C})$ Yang-Mills fields. [ $P$ would be subject to certain algebraic constraints and/or reality conditions when the gauge group is a subgroup of $S L(n, \mathbb{C}) ; P$ would also be subject to further conditions if we required global solutions.] Conversely starting with a general ASD solution to the YangMills equations, we can always produce a $P$ by solving 2.9 a with $G_{0}(x, \zeta)$ for $|\zeta|<\infty$, and $G_{\infty}(x, \zeta)$ for $|\zeta|>0$, and then put $P=G_{\infty}^{-1} G_{0}$.

The patching matrix $P$ is given up to the "gauge" freedom

$$
P^{\prime}(L, \bar{M}, \zeta)=N^{-1}(L, \bar{M}, \zeta) P(L, \bar{M}, \zeta) S(L, M, \zeta),
$$

where $S$ and $N$ are non-singular $n \times n$ matrices of functions holomorphic in $U_{0}$ and $U_{\infty}$ respectively; the matrix $P^{\prime}$ being equivalent to $P$. This transformation can be seen to generate a "right-gauge" transformation on the matrix-valued functions 
$G_{0}$ and $G_{\infty}$ obtained from the splitting, (2.12), of $P^{\prime}$,

and

$$
G_{0}^{\prime}\left(x^{a}, \zeta\right)=G_{0}\left(x^{a}, \zeta\right) S(L, \bar{M}, \zeta)
$$

$$
G_{\infty}^{\prime}\left(x^{a}, \zeta\right)=G_{\infty}\left(x^{a}, \zeta\right) N(L, \bar{M}, \zeta) .
$$

Note that this right-gauge transformation has no effect on the ASDYM connection $\gamma_{a}$, defined in $(2.13 \mathrm{a}, \mathrm{b})$, i.e. the $\gamma_{a}$ constructed from the $G$ is the same as that constructed from the $G^{\prime}$.

A different type of gauge transformation, namely left-multiplication of the $G_{0}$ and $G_{\infty}$ by a non-singular $n \times n$ matrix-valued functions of $x^{a}$, can be defined. Such left-gauge transformations, given by

and

$$
G_{0}^{\prime}\left(x^{a}, \zeta\right)=C\left(x^{a}\right) G_{0}\left(x^{a}, \zeta\right)
$$

$$
G_{\infty}^{\prime}\left(x^{a}, \zeta\right)=C\left(x^{a}\right) G_{\infty}\left(x^{a}, \zeta\right),
$$

do not alter the patching matrix $P$, i.e. they represent the freedom in the solution of the splitting problem. They correspond to ordinary gauge transformations on the ASDYM connection $\gamma_{a},(2.13 \mathrm{a}, \mathrm{b})$.

These transformations can be fixed by specifying the value of $G_{0}\left(x^{a}, \zeta\right)$ or $G_{\infty}\left(x^{a}, \zeta\right)$ at some $\zeta=\zeta_{1}$ for each value of $x^{a}$ (effectively fixing the matrix $C$ and the gauge for $\gamma_{a}$ ). Such a procedure, is referred to as left-gauge fixing. A common choice is to set $G_{\infty}\left(x^{a}, \infty\right)=I$. The quantity $G_{0}\left(x^{a}, 0\right)$ then turns out to determine the entire connection of the solution. It is often referred to as Yang's J-matrix [2].

\section{Splitting of a Triangular Patching Matrix}

In this section a procedure is given for solving the $n \times n$ matrix-valued splitting Eq. (2.12) to obtain $G_{0}$ and $G_{\infty}$, for a patching matrix $P$ that is of triangular form. This procedure generalizes the $2 \times 2$ version of Corrigan et al. $[3,11]$.

Note that $P$ is a given function of the arguments $(L, \bar{M}, \zeta)$, but in the splitting problem, we restrict the values of $L, \bar{M}$ via (2.10), so that $P$ becomes a function of $x^{a}$ and $\zeta$. Consider an upper triangular $S L(n, \mathbb{C})$ patching matrix $P=\left[p_{i j}\right]$, i.e.,

and

$$
p_{i j}=0 \text { for } i>j
$$

$$
\operatorname{det} P=1 \text {. }
$$

Remark 3. A $G L(n, \mathbb{C})$ matrix could be considered, but such a matrix can be factorized into an $S L(n, \mathbb{C})$ matrix and the determinant part, whose splitting is accomplished by solving a scalar RH problem. Therefore only $S L(n, \mathbb{C})$ matrices need to be considered.

The diagonal elements of $P$ can be expressed as

$$
p_{i i}=\zeta^{\alpha_{i}} e^{f_{i}}
$$

with $\operatorname{det} P=1$ equivalent to

$$
\sum_{i} \alpha_{i}=0 \quad \text { and } \quad \sum_{i} f_{i}=0 .
$$


For each $i$, the integer $\alpha_{i}$, the winding number or index of the function $p_{i i}$, is the number of times the value of the function winds about the origin, as $\zeta$ moves around the annulus $U_{0} \cap U_{\infty}$. The winding number is given by

$$
\alpha_{j}=\frac{1}{2 \pi} \oint_{C} \frac{p_{j j, \zeta} d \zeta}{p_{j j}}
$$

or

$$
\alpha_{j}=\frac{1}{2 \pi i}\left[\log p_{j j}\right]_{c}
$$

where $C$ specifies a closed loop linking the annulus once and []$_{c}$ denotes the increment of the expression in the brackets as a result of a circuit around $C$. The functions $f_{i}$ have zero winding number and are single-valued functions on the annulus. The form of the decomposition of the diagonal elements of the matrix given in (3.2) assumed that the functions $p_{i i}$ contained no zeros in the annulus; this follows from the condition $\operatorname{det} P=1$ and the fact that $P$ is upper triangular, so that $\operatorname{det} P$ is just the product of its diagonal entries.

Remark 4. It is worth noting that in order to split the upper triangular matrix $P$, the following conditions must be satisfied:

$$
\sum_{i=j}^{n} \alpha_{i} \leqq 0 \quad j \in\{1, \ldots, n\} .
$$

If these conditions are not satisfied then the Birkhoff decomposition for a triangular matrix will always have $H(\zeta) \neq 1$. Roughly speaking this is because the lower right $n-j \times n-j$ block of the matrix is the patching matrix of a subbundle of the full bundle, and when this inequality fails, this subbundle has positive Chern class. On restriction to a Riemann sphere, this subbundle will then have more than $n-j$ independent sections. This means that the bundle is nontrivial on restriction to spheres, so that when split, the patching matrix will always yield non-trivial $H$. Generically, if these conditions are satisfied we will have that $H(\zeta)=1$. Note that the above inequality and the $\operatorname{det} P=1$ condition ensures that $\alpha_{1}$ is positive.

Before splitting the matrix $P$, we split the functions $f_{i}$ into the functions $f_{i}^{0}$ and $f_{i}^{\infty}$, holomorphic in $U_{0}$ and $U_{\infty}$ respectively, such that (on the annulus)

$$
f_{i}=f_{i}^{0}-f_{i}^{\infty} .
$$

The functions $f_{i}^{0}$ and $f_{i}^{\infty}$ will be used extensively in the splitting of the matrix $P$ which follows. This splitting of $f_{i}$ is an example of a scalar $\mathrm{RH}$ problem and is given by

$$
f_{i}^{\infty}=\frac{1}{2 \pi i} \oint_{c} \frac{f_{i} d \zeta^{\prime}}{\zeta^{\prime}-\zeta} \text { and } f_{i}^{0}=\frac{1}{2 \pi i} \oint_{c} \frac{f_{i} \mathrm{~d} \zeta^{\prime}}{\zeta^{\prime}-\zeta^{\prime}},
$$

where the integral is taken along a closed contour $C$ in the annulus $U_{0} \cap U_{\infty}$ with $\zeta \in U_{\infty}$ for $f_{i}^{\infty}$ and $\zeta \in U_{0}$ for $f_{i}^{0}$. The expressions (3.7) are obtained from a direct application of the Cauchy integral theorem to (3.6).

The splitting problem, (2.12), if written out with $P$ in upper triangular form, 
yields $n^{2}$ scalar RH problems, $n$ of which are homogeneous problems for the diagonal entries and the remaining $n(n-1)$ equations form a hierarchy of inhomogeneous RH problems. Each step in the hierarchy can be solved by quadratures using the results discussed previously in Remark 1, once the preceding steps have been solved. The results are now summarized.

Denoting the elements of the matrix-valued functions $G_{0}$ and $G_{\infty}$ by $g_{i j}^{0}$ and $g_{i j}^{\infty}$ respectively, we write the solutions to the $n$ homogeneous RH equations as

$$
g_{i 1}^{0}=e^{-f_{1}^{0}} \sum_{s=0}^{\alpha_{1}} A_{i s} \zeta^{s}
$$

and

$$
g_{i 1}^{\infty}=e^{-f_{1}^{\infty}} \sum_{s=0}^{\alpha_{1}} A_{i s} \zeta^{s-\alpha_{1}},
$$

with $A_{\text {is }}$ for the moment being arbitrary functions of $x$. We give the ansatze for the solutions to the $n(n-1)$ inhomogeneous RH equations (for $j \neq 1$ )

$$
g_{i j}^{0}=e^{-f_{j}^{0}} \frac{1}{2 \pi i} \oint_{c} \frac{e^{-f_{j}^{0}} \sum_{h<j} g_{i h}^{\infty} p_{h j} d \zeta^{\prime}}{\left(\zeta^{\prime}-\zeta\right)}
$$

and

$$
g_{i j}^{\infty}=e^{-f_{j}^{\infty}} \zeta^{-\alpha_{j}} \frac{1}{2 \pi i} \oint_{c} \frac{e^{-f_{j}^{0}} \sum_{h<j} g_{i h}^{\infty} p_{h j} d \zeta^{\prime}}{\left(\zeta^{\prime}-\zeta\right)},
$$

where the integral is taken along a closed contour $C$ linking the annulus $A$ once. For the first integral $\zeta$ lies on the zero side of the contour and for the second $\zeta$ lies on the infinity side of the contour. This ansatze does not solve the RH-problem unless the following quantities vanish:

$$
\Omega_{i j s}=\frac{1}{2 \pi i} \oint_{c} \frac{e^{-f_{j}^{0}} \sum_{h<j} g_{i h}^{\infty} p_{h j} d \zeta}{\zeta^{s+1}},
$$

for $0>s>\alpha_{i}$.

These conditions result from the fact that if the $\Omega_{i j s}$ for $0>s>\alpha_{i}$ do not vanish, the inhomogeneous RH problem is obstructed as in Remark 1. The $\Omega_{i j s}$ depend on the choice of $A_{i s}$ and by varying this excess freedom in the homogeneous problem we can (generally) make these obstructions vanish. When $\alpha_{i}>0$, there is again excess freedom in the solution to the problem, but this will in general have to be used to put further obstructions equal to zero. Counting the number of free functions and the number of conditions in (3.9), it can be seen that (3.9) fixes the freedom in the choice of the functions $A_{\text {is }}$ to the point where there are only $n^{2}-1$ free functions to choose. This remaining freedom just corresponds to the general $S L(n, \mathbb{C})$ gauge freedom.

It is not always possible to eliminate the obstructions. There are however nondegeneracy conditions on certain components of the Laurent series for the off 
diagonal $p_{i j}$ that, when satisfied, guarantee that the freedom in the homogeneous problem can indeed be used to set the obstructions in (3.10) equal to zero. These conditions are referred to as the catalecticant (or simplex) conditions $[18,19]$. If these conditions fail at some points, the Yang-Mills field will be singular at those points. Generically they will not fail.

Having obtained $G_{0}$ and $G_{\infty}$, the corresponding $S L(n, \mathbb{C})$ anti-self-dual connection $\gamma_{a}$ can now be calculated according to $(2.13 \mathrm{a}, \mathrm{b})$.

\section{Triangularization of the $S L(2, \mathbb{C})$ Patching Matrix}

We shall delay the approximation and triangularization of an arbitrary $S L(n, \mathbb{C})$ patching matrix $P$ until $V I$ and first analyze the $S L(2, \mathbb{C})$ case. We show how an arbitrary $S L(2, \mathbb{C})$ patching matrix $P$ can be approximated, with arbitrary accuracy, such that each approximate patching matrix can be put into upper triangular form so that it can be split and used to construct an exact ASDYM connection and field which approximates the field of the original data.

Consider first a general $S L(2, \mathbb{C})$ patching matrix $P$. We wish to transform it, via (2.14), to $P_{T}$ which is upper triangular. Choosing $N(L, \bar{M}, \zeta)=1$, the transformed patching matrix is given by

$$
P_{T}(L, \bar{M}, \zeta)=P(L, \bar{M}, \zeta) S(L, \bar{M}, \zeta)
$$

The condition that $P_{T}$ be upper triangular leads to one equation for the two components, $s_{11}$ and $s_{21}$ of $S$ in terms of the two components $p_{21}$ and $p_{22}$ of $P$, namely

$$
p_{21} s_{11}+p_{22} s_{21}=0
$$

where $s_{11}$ and $s_{21}$ are to be holomorphic in $U_{0}$, while $p_{21}$ and $p_{22}$ are holomorphic in $U_{0} \cap U_{\infty}$. This expression can be rewritten as

$$
\frac{p_{21}}{p_{22}}=-\frac{s_{21}}{s_{11}}
$$

The ratio $p_{21} / p_{22}$ is meromorphic on $U_{0} \cap U_{\infty}$ whereas the ratio $s_{21} / s_{11}$ is to be meromorphic on $U_{0}$. Obtaining such a ratio $s_{21} / s_{11}$ for general functions $p_{21}$ and $p_{22}$ is not possible. (Note that if $p_{21} / p_{22}$ were meromorphic on $U_{0}$, then (4.3) could be solved for the components $s_{11}$ and $s_{21}$ ). When $p_{21} / p_{22}$ is not meromorphic on $U_{0}$, the ratio $p_{21} / p_{22}$ can still be approximated by a sequence of meromorphic functions on $U_{0}$, namely $\left(p_{21}\right) /\left(p_{22}\right)^{(m)}$, (thereby effictively approximating one of the two components $p_{21}$ or $p_{22}$ and so approximating the general patching matrix $P$ by the approximate patching matrix $P^{(m)}$ ). A solution for $s_{11}$ and $s_{21}$ can be explicitly obtained for the approximate $P^{(m)}$. The remainder of this section details the approximation scheme for $S L(2, \mathbb{C})$ patching matrices and the triangularization procedure for the approximate patching matrix $P^{(m)}$.

We first define a closed annular subset $U_{0}^{r} \cap U_{\infty}^{r}$ of the full annulus $U_{0} \cap U_{\infty}$ as the intersection of the two closed patches $U_{0}^{r}$ and $U_{\infty}^{r}$ which cover PT defined by

$$
U_{0}^{r}=\left\{\mathbb{P T} /|\zeta| \leqq \frac{1}{r}\right\} \quad \text { and } \quad U_{\infty}^{r}=\{\mathbb{P T} /|\zeta| \leqq r\} \text {, }
$$


for $0 \leqq r<1$. The annulus $U_{0}^{r} \cap U_{\infty}^{r}$ (with $U_{0}^{r}$ and $U_{\infty}^{r}$ as defined above) is a closed annular subset of $U_{0} \cap U_{\infty}$. This change does not affect either the twistor construction described in chapter III or the triangularization condition (4.2) or (4.3), but does affect convergence arguments for the approximation procedure which follows.

We now describe the approximation procedure for the ratio $p_{21} / p_{22}$. From the Mittag-Leffler theorem [20], the ratio $p_{21} / p_{22}$ can be expressed on $U_{0}^{r} \cap U_{\infty}^{r}$ as

$$
\frac{p_{21}(L, \bar{M}, \zeta)}{p_{22}(L, \bar{M}, \zeta)}=\sum_{i} \frac{b_{i}(L, \bar{M})}{\left(\zeta-\zeta_{i}(L, \bar{M})\right)^{\alpha_{i}}}+\sum_{n=-\infty}^{+\infty} a_{n}(L, \bar{M}) \zeta^{n}
$$

where all the poles of $p_{21} / p_{22}$ in $U_{0}^{r} \cap U_{\infty}^{r}$ are at $\zeta=\zeta_{i}$ (these poles are of order $\alpha_{i}$ ) and the second term on the right-hand-side (the Laurent series, $\left.\sum_{n} a_{n} \zeta^{n}\right)$ is holomorphic on $U_{0}^{r} \cap U_{\infty}^{r}$. Note that the zeros of $p_{22}$ are at $\zeta=\zeta_{i}$ and of order $\alpha_{i}$ (the zeros of $p_{22}$ define the poles of $\left.p_{21} / p_{22}\right)$ and that the $b_{i}(L, \bar{M})$ 's are non-zero at $\zeta=\zeta_{i}$ because $p_{21}$ and $p_{22}$ do not contain common zeros (otherwise the patching matrix $P$ would be singular on $U_{0} \cap U_{\infty}$ ).

Remark 5. The set of functions $a_{n}$ are analytic functions of the variables $L, \bar{M}$ in the domain under consideration, which will be $U_{0}^{r}$ with $L$ and $\bar{M}$ in some neighbourhood of zero. This can be seen from the fact that our considerations are local in $\mathbb{M}$ and can be taken in the neighborhood of $x^{a}=0$ so that since $L=L_{a}(\zeta) x^{a}$ and $\bar{M}=\bar{M}_{a}(\zeta) x^{a}$, which are linear in $\zeta$, gives a neighbourhood of $L=\bar{M}=0$ in $U_{0}^{r}$. Thus the $a_{n}$ 's will be analytic in the neighborhood of $L, \bar{M}=0$. This point becomes important later when the $a_{n}$ 's have to be evaluated on $U_{0}^{r}$.

The ratio $p_{21} / p_{22}$ will be uniformly approximated by

$$
\left(\frac{p_{21}(L, \bar{M}, \zeta)}{p_{22}(L, \bar{M}, \zeta)}\right)^{(m)}=\sum_{i} \frac{b_{i}(L, \bar{M})}{\left(\zeta-\zeta_{i}(L, \bar{M})\right)^{\alpha_{2}}}+\sum_{n=-m}^{+m} a_{n}(L, \bar{M}) \zeta^{n}+\frac{1}{2^{m+1}} \frac{1}{\zeta^{m+1}}
$$

where the $a_{n}(L, \bar{M})$ 's are the same functions are given in (4.5). The Laurent series in (4.5) is approximated by the partial sum in (4.6), and such a Laurent series is uniformly convergent on the closed annulus $U_{0}^{r} \cap U_{\infty}^{r}$ [21]. Because we are working on the closed annulus and the Laurent series in (4.5) is uniformly convergent, the ratio $\left(p_{21} / p_{22}\right)^{(m)}$ can approximate the general ratio $p_{21} / p_{22}$ as closely as desired by varying the limit $m$ of the partial sum [22]. Also note that the approximated ratio uniformly converges to $(4.5)$ as $m \rightarrow \infty$. The term $\left(1 / 2^{m+1}\right) /\left(1 / \zeta^{m+1}\right)$ has been added to the partial sum to assure that the coefficient of the lowest power of $\zeta$, the $-(m+1)$ power, is non-zero; this condition is not required for the approximation schemes but is important in triangularizing the approximate patching matrix, and will be more fully explained later.

The triangularization condition (4.3) now becomes

$$
\left(\frac{p_{21}}{p_{22}}\right)^{(m)}=-\frac{s_{21}}{s_{11}}
$$

Given the expression (4.6) for the ratio $\left(p_{21} / p_{22}\right)^{(m)}$, the triangularization condition 
can easily be satisfied by letting

$$
s_{11}(L, \bar{M}, \zeta)=\zeta^{m+1} \prod_{i}\left(\zeta-\zeta_{i}(L, \bar{M})\right)^{\alpha_{i}}
$$

and

$$
\begin{aligned}
s_{21}(L, \bar{M}, \zeta)= & \zeta^{m+1} \sum_{i} b_{i}(L, \bar{M}) \prod_{k \neq i}\left(\zeta-\zeta_{k}(L, \bar{M})\right)^{\alpha_{k}} \\
& +\prod_{i}\left(\zeta-\zeta_{i}(L, \bar{M})\right)^{\alpha_{i}}\left[\sum_{n=-m}^{+m} a_{n}(L, \bar{M}) \zeta^{n+m+1}+\frac{1}{2^{m+1}}\right] .
\end{aligned}
$$

The components $s_{11}$ and $s_{21}$ are polynomials in positive powers of $\zeta$ and are therefore holomorphic in $U_{0}^{r}$. It is important that the components $s_{11}$ and $s_{21}$ do not contain common zeros; $s_{11}$ vanishes at $\zeta=0$ and $\zeta=\zeta_{i}$ but $s_{21}$ does not vanish at $\zeta_{i}$, since the $b_{i}$ 's are non-zero, and does not vanish at $\zeta=0$ by virtue of the added factor $1 / 2^{m+1}$.

Having obtained expressions for $s_{11}$ and $s_{21}$, the other elements of the transformation matrix $S$, namely $s_{12}$ and $s_{22}$, must be determined. Imposing $\operatorname{det} S=1$ on the transformation matrix not only assures that $S$ will be non-singular, but also provides the condition by which the elements $s_{12}$ and $s_{22}$ can be determined. Note that it is possible to satisfy the determinant condition on $U_{0}^{r}$ only because $s_{11}$ and $s_{21}$ do not simultaneously vanish.

Since the functions $s_{11}$ and $s_{21}$ are polynomials in positive powers of $\zeta$, they can be expanded as

$$
s_{11}=\sum_{n=0}^{2 m+\alpha+1} s_{11 n}(L, \bar{M}) \zeta^{n}
$$

and

$$
s_{21}=\sum_{n=0}^{2 m+\alpha+1} s_{21 n}(L, \bar{M}) \zeta^{n}
$$

where $\alpha=\sum_{i} \alpha_{i}$. Some of the coefficients, $s_{11 n}$ and $s_{21 n}$, may be żero, but it is important to note that $s_{210} \neq 0$ (since $s_{21}$ does not vanish at $\zeta=0$ ). The other two components, $s_{12}$ and $s_{22}$ are assumed to have the expansions

$$
s_{12}=\sum_{n=0}^{2 m+\alpha+1} s_{12 n}(L, \bar{M}) \zeta^{n}
$$

and

$$
s_{22} \sum_{n=0}^{2 m+\alpha+1} s_{22 n}(L, \bar{M}) \zeta^{n} .
$$

The determinant condition on $S, s_{11} s_{22}-s_{12} s_{21}=1$, can be viewed as defining relations on the coefficients $s_{12 n}$ and $s_{22 n}$ in terms of $s_{11 n}$ and $s_{11 n}$. These relations

$$
\sum_{q=0}^{n}\left(s_{11 q} s_{22(n-q)}-s_{21 q} s_{12(n-q)}\right)=\delta_{0}^{n}, \quad n \in\{0, \ldots, 2 m+\alpha+1\}
$$


from a set of non-homogeneous linear algebraic equations which can be written in matrix form as

$$
B X=C,
$$

where $B$ is a $(4 m+2 \alpha+4) \times(2 m+\alpha+2)$ matrix of the coefficients of $s_{11}$ and $s_{21}$, $X$ is a column matrix of the coefficients of $s_{12}$ and $s_{22}$, and $C$ is a column matrix of constants. The matrices $B, X$, and $C$ can be written

$$
\begin{aligned}
& B_{i j}=\left\{\begin{array}{l}
s_{11 n}, n=i-\frac{j}{2} \text { for } j \text { even or } 0 \\
-s_{21 n}, n=i-\frac{(j-1)}{2} \text { for } j \text { odd }
\end{array}\right\}, \\
& X_{i}=\left\{\begin{array}{l}
s_{22 i}, \text { for } i \text { even or } 0 \\
s_{12 \frac{(i-1)}{2}}, \text { for } i \text { odd }
\end{array}\right\} . \\
& C_{i}=\delta_{i}^{0} .
\end{aligned}
$$

This set of algebraic equations has a non-trivial (but not necessarily unique) solution as long as $B_{0 i} \neq 0[23]$ for some $i$. Note that an underdetermined system of algebraic equations has a non-trivial solution when the rank of the coefficient matrix (the matrix $B$ ) is the same as the rank of the augmented coefficient matrix (the matrix $B$ with the column $C$ added). This follows since $B_{01}=-s_{210} \neq 0$ (again the importance of the $\left(1 / 2^{m+1}\right) /\left(1 / \zeta^{m+1}\right)$ term in the partial sum (4.6) can be seen; its addition ensures that the set of algebraic equation (4.11) has a non-trivial solution). Therefore, given the functions $s_{11}$ and $s_{21}$, functions $s_{12}$ and $s_{22}$ can be found, yielding a non-singular transformations matrix $S$.

Remark 6. An important point is that the construction of the transformation matrix $S$ will diverge in the limit $m \rightarrow \infty$ (since the elements $s_{12}$ and $s_{22}$ contain factors of $2^{m+1}$ ). Therefore the triangularization of a general patching matrix $P$, obtained by taking the limit $m \rightarrow \infty$ of (4.6), cannot be accomplished by this method.

Remark 7. A more abstract argument for the existence of the elements $s_{12}$ and $s_{22}$ is the following. The construction of the functions $s_{11}$ and $s_{21}$ produces a line subbundle $L$ of $E$ which consists of vectors proportional to $\left(\begin{array}{l}1 \\ 0\end{array}\right)$ on $U_{\infty}$ and vectors proportional to $\left(\begin{array}{l}s_{11} \\ s_{21}\end{array}\right)$ on $U_{0}$. This means that a line bundle $M\left(=L^{*}\right)$ can be defined to be the quotient $E / L$. On $U_{0}$ all bundles are trivial and so a non-vanishing section of $M$ can be found. This is an equivalence class of sections of $E$ modulo sections of $L$. Since $U_{0}$ is analytically trivial, a representative element of this equivalence class $\left(\begin{array}{l}s_{12} \\ s_{22}\end{array}\right)$ exists on all of $U_{0}$ and the matrix $S$ is thus obtained. [It may be necessary to rescale this section so that $S$ has unit determinant].

Having obtained the transformation matrix $S$, the triangular patching matrix 
$P_{T}^{(m)}$ which is equivalent to the approximate patching matrix $P^{(m)}$ becomes

$$
P_{T}^{(m)}(L, \bar{M}, \zeta)=P^{(m)}(L, \bar{M}, \zeta) S(L, \bar{M}, \zeta)
$$

This triangular patching matrix can now be split by the procedure described in Sect. III.

\section{Convergence of the Approximate Solution}

The triangularization procedure outlined yields a patching matrix $P^{(m)}$ which is an approximation to the general patching matrix $P$. Each $P^{(m)}$ can be split to yield $G_{0}^{(m)}$ and $G_{\infty}^{(m)}$, and then by differentiation, a solution $\gamma_{a}^{(m)}$ of the ASDYM equations. We shall assume that for the original $P$ we have a regular splitting over some compact domain in space-time, so that there are no jumping lines for the original $P$ :

$$
G_{\infty}\left(x^{a}, \zeta\right) P(L, \bar{M}, \zeta)=G_{0}\left(x^{a}, \zeta\right),
$$

and generically we will also have

$$
G_{\infty}^{(m)}\left(x^{a}, \zeta\right) P^{(m)}(L, \bar{M}, \zeta)=G_{0}^{(m)}\left(x^{a}, \zeta\right) .
$$

The question arises as to how closely the split solutions $G_{0}^{(m)}$ and $G_{\infty}^{(m)}$ obtained from the patching matrix $P^{(m)}$ approximate $G_{0}$ and $G_{\infty}$ obtained from the patching matrix $P$, and how closely the connection $\gamma_{a}^{(m)}$ obtained from $G_{0}^{(m)}$ and $G_{\infty}^{(m)}$ approximates $\gamma_{a}$ obtained from $G_{0}$ and $G_{\infty}$.

Firstly in order to obtain convergence for $G_{0}^{(m)}$ and $G_{\infty}^{(m)}$, we must fix the gauge ambiguity by setting $G_{\infty}^{(m)}(x, \infty)=I=G_{\infty}(x, \infty)$. The approximate split solutions $G_{0}^{(m)}$ and $G_{\infty}^{(m)}$ can then be seen to converge respectively to $G_{0}$ and $G_{\infty}$ as a consequence of the fact that the map from the patching data to the splitting functions is continuous. More formally, in ref. [17], in Proposition 1.1, pp. 205, it is proven that if $P=G_{0} G_{\infty}$ then for all $\varepsilon>0$, there exists a $\delta$ such that $\left\|P-P^{(m)}\right\|_{\infty}<\delta$ implies that

$$
\left\|G_{0}^{(m)}-G_{0}\right\|_{\infty}<\varepsilon \text { and }\left\|G_{\infty}^{(m)}-G_{\infty}\right\|_{\infty}<\varepsilon
$$

together with the same inequalities for their inverses. This theorem is stated in the context of just one RH problem. It is clear that if the domain in space-time is taken to be compact, then there is no difficulty in finding a $\delta$ valid for the whole region.

In Proposition 1.2 on pp. 206 of [17] it is proved that if $P$ depends analytically on some parameters, in this case the space-time coordinates, then the $G$ 's also depend analytically on the parameters. The same also goes for the $P^{(m)}$ 's. Therefore we have a sequence of analytic functions of $x$, and $\zeta, G_{0}^{(m)}$ and $G_{\infty}^{(m)}$ converging on $G_{0}$ and $G_{\infty}$ in the \|\|$_{\infty}$ norm. The analyticity implies that if the functions converge, then their derivatives also converge. Thus, since the connection $\gamma_{a}^{(m)}$ which approximates $\gamma_{a}$, is obtained from the derivatives of $G_{0}^{(m)}$ from (2.13), it converges to $\gamma_{a}$ as the approximate patching matrix $P^{(m)}$ converges to $P$.

The arguments given in this section apply to any $S L(n, \mathbb{C})$ patching matrix. 


\section{Triangularization of the $S L(n, \mathbb{C})$ Patching Matrix}

In Sect. IV the approximation and triangularization of an $S L(2, \mathbb{C})$ patching matrix was discussed; in this section, this procedure is generalized to the $S L(n, \mathbb{C})$ case.

Consider a general $S L(n, \mathbb{C})$ patching matrix $P$. We shall attempt to transform this patching matrix, as in (4.1), to $P_{T}$ which is upper triangular; the condition that $P_{T}$ be upper triangular leads to a set of $(n(n-1) / 2)$ equations for the components of $S, s_{j k}$, in terms of the components of $P, p_{j k}$. The triangularization conditions are

$$
p_{j k}^{T}=\sum_{h=1}^{n} p_{j h} s_{h k}=0 \text { for } k<j,
$$

where the $s_{j k}$ 's are holomorphic in $U_{0}$ (or $U_{0}^{r}$ ), the $p_{j k}$ 's and $p_{j k}^{T}$ 's are holomorphic in $U_{0} \cap U_{\infty}$ (or $U_{0}^{r} \cap U_{\infty}^{r}$ ), and the $p_{j k}^{T}$ 's are the elements of the triangular $P_{T}$. In general, these triangularization equations have no solution. Nevertheless the general patching matrix $P$ can be approximated by patching matrices $P^{(m)}$ for which the equations can be solved. The remainder of this section outlines a procedure by which a generic $S L(n, \mathbb{C})$ patching matrix may be approximated and this approximate patching matrix may be triangularized.

It has been shown in Sect. IV that a general $S L(2, \mathbb{C})$ patching matrix may be approximated and that this approximate patching matrix may be triangularized. In order to show that a general $S L(n, \mathbb{C})$ patching matrix may be approximated and triangularized, assume that such a procedure exists for a general $S L(n-1, \mathbb{C})$ patching matrix. It will then be shown that the triangularization conditions, (6.1), for the general $S L(n, \mathbb{C})$ patching matrix reduce to two sets of equations. For the first set of equations, an approximation scheme and solution will be developed. The second set of equations is equivalent to the triangularization equations for an $S L(n-1, \mathbb{C})$ patching matrix, which is soluble by assumption. This completes the inductive argument for the triangularization of the $S L(n, \mathbb{C})$ patching matrix.

Begin by assuming that a general $S L(n-1, \mathbb{C})$ patching matrix $(n>2)$ can be approximated and triangularized as before. The triangularization transformation (4.1) for an $S L(n, \mathbb{C})$ patching matrix $P$ can be rewritten as

$$
S(L, \bar{M}, \zeta)=P^{-1}(L, \bar{M}, \zeta) P_{T}(L, \bar{M}, \zeta),
$$

where $P_{T}$ is upper triangular. In terms of components, the matrix $S$ is given by

$$
s_{i j}(L, \bar{M}, \zeta)=\sum_{k=1}^{n} \Delta_{i k}(L, \bar{M}, \zeta) p_{k j}^{T}(L, \bar{M}, \zeta) .
$$

where the $\Delta_{k i}$ 's are the cofactors of the patching matrix $P$ and the $p_{k j}^{T}$ 's are the components of the upper triangular patching matrix $P_{T}\left(p_{k j}^{T}=0\right.$ for $\left.j<k\right)$. Because $P_{T}$ is upper triangular, Eq. (6.3) for $s_{i 1}$ is particularly simple,

$$
s_{i 1}(L, \bar{M}, \zeta)=\Delta_{i 1}(L, \bar{M}, \zeta) p_{11}^{T}(L, \bar{M}, \zeta) .
$$

In keeping with the spirit of Sect. IV, consider ratios of these components of the form:

$$
\frac{s_{i 1}}{s_{11}}=\frac{\Delta_{i 1}}{\Delta_{11}}
$$


This set of expressions is the analogue of the expression (4.3) for $n=2$ (for $n=2$, we have $\Delta_{21}=-p_{21}$ and $\Delta_{11}=p_{22}$ ). The ratios $\Delta_{i 1} / \Delta_{11}$ are meromorphic on the closed annulus $U_{0}^{r} \cap U_{\infty}^{r}$ and so can also be expressed in a form similar to (4.5), namely

$$
\frac{\Delta_{i 1}(L, \bar{M}, \zeta)}{\Delta_{11}(L, \bar{M}, \zeta)}=\sum_{k} \frac{b_{k}^{(i)}(L, \bar{M})}{\left(\zeta-\zeta_{k}(L, \bar{M})\right)^{\alpha_{k}}}+\sum_{n=-\infty}^{+\infty} a_{n}^{(i)}(L, \bar{M}) \zeta^{n}
$$

where all the poles of $\Delta_{i 1} / \Delta_{11}$ in $U_{0}^{r} \cap U_{\infty}^{r}$ are at $\zeta=\zeta_{k}$ (of order $\alpha_{k}$ ) and do not depend on $i$ since these poles correspond to the zeros of $\Delta_{11}$. The second term on the right-hand-side of (6.6) (the Laurent series, $\left.\sum_{n} a_{n}^{(i)} \zeta^{n}\right)$ is holomorphic on $U_{0}^{r} \cap U_{\infty}^{r}$. It is important to note that the $b_{k}^{(i)}$ 's cannot all simultaneously vanish at $\zeta=\zeta_{k}$, otherwise, the patching matrix would be singular. Again the functions $a_{n}^{(i)}$ are to be considered analytic functions of the variables $L$ and $\bar{M}$.

These ratios can now be uniformly approximated by

$$
\left(\frac{\Delta_{1 i}}{\Delta_{11}}\right)^{(m)}=\sum_{k} \frac{b_{k}^{(i)}(L, \bar{M})}{\left(\zeta-\zeta_{k}(L, \bar{M})\right)^{\alpha_{k}}}+\sum_{n=-m}^{+m} a_{n}^{(i)}(L, \bar{M}) \zeta^{n}+\frac{1}{2^{m+1}} \frac{1}{\zeta^{m+1}},
$$

where the $a_{n}^{(i)}(L, \bar{M})$ 's are the same functions are given in (6.6). These approximated ratios uniformly converge to $(6.6)$ as $m \rightarrow \infty$ and so the ratio $\left(\Delta_{1 i} / \Delta_{11}\right)^{(m)}$ can approximate the general ratio $\Delta_{i 1} / \Delta_{11}$ as closely as desired by varying the limit $m$ of (6.7) [22]. Again, the term $\left(1 / 2^{m+1}\right) /\left(1 / \zeta^{m+1}\right)$ has been added to the partial sum to assure that the transformation matrix $S$, defined from these approximated ratios, is non-singular.

With the ratios $\Delta_{i 1} / \Delta_{11}$ approximated in the above manner, (6.5) becomes

$$
\left(\frac{\Delta_{1 i}}{\Delta_{11}}\right)^{(m)}=\frac{s_{i 1}}{s_{11}}
$$

Given the expressions $(6.7)$ for the ratios $\left(\Delta_{1 i} / \Delta_{11}\right)^{(m)}$, the elements of the transformation matrix, $s_{i 1}$, can be determined, yielding

$$
s_{11}(L, \bar{M}, \zeta)=\zeta^{m+1} \prod_{k}\left(\zeta-\zeta_{k}(L, \bar{M})\right)^{\alpha_{k}}
$$

and for $i \neq 1$,

$$
\begin{aligned}
s_{i 1}(L, \bar{M}, \zeta)= & \zeta^{m+1} \sum_{k} b_{k}^{(i)}(L, \bar{M}) \prod_{q \neq k}\left(\zeta-\zeta_{q}(L, \bar{M})\right)^{\alpha_{q}} \\
& +\prod_{k}\left(\zeta-\zeta_{k}(L, \bar{M})\right)^{\alpha_{k}}\left[\sum_{n=-m}^{+m} a_{n}^{(i)}(L, \bar{M}) \zeta^{n+m+1}+\frac{1}{2^{m+1}}\right]
\end{aligned}
$$

The components of $s_{i 1}$ are polynomials in positive powers of $\zeta$ and so are holomorphic in $U_{0}^{r}$. Note that the components $s_{i 1}$ cannot all simultaneously vanish. $\left(s_{11}\right.$ vanishes at $\zeta=0$ and $\zeta=\zeta_{k}$ but the $s_{i 1}$ 's $(i \neq 1)$ do not vanish at $\zeta=0$ by virtue of the added factor $1 / 2^{m+1}$ and cannot all vanish at $\zeta=\zeta_{k}$ since the $b_{k}^{(i)}$ 's cannot all simultaneously vanish.) The expressions (6.9) determine the first column of the 
transformation matrix $S$; the explicit construction of the $s_{i 1}$ 's insures (at this stage at least) that $S$ is non-singular.

We have approximated the patching matrix $P$ with $P^{(m)}$ in such a way as to obtain the first column of the transformation matrix $S$. Care was exercised in order to ensure that this column vanished nowhere. We can now choose the subsequent column of $S$ in such a way as to guarantee that the matrix $S$ in nonsingular with unit determinant on $U_{0}$. This follows from a generalization of either the abstract or the concrete argument at the end of Sect. IV. The abstract argument follows by considering the column vector as a nonvanishing section of the vector bundle on $U_{0}$, and by choosing a frame for the quotient of the bundle by this section on $U_{0}$ (which must exist since $U_{0}$ is Stein). This frame gives $(n-1)$ independent sections of the vector bundle on $U_{0}$ which are also independent of the first column of $S$, and so constitute a frame. The components of this frame are the entries of $S$. The concrete argument followed by expanding out the undetermined entries of $S$ in a power series, and solving for them subject to the condition that $S$ has unit determinant, and showing that there exists a solution; cf. Eq. (4.11) and the arguments applied to it.

Using this $S$, we can gauge transform $P^{(m)}$ so that the first column of $P^{(m)} S$ is zero apart from $\left(P^{(m)} S\right)_{11}$. Effectively $n-1$ of the triangularization equations $(6.1)$ (for the approximate patching matrix $P^{(m)}$ ) have been solved and there remain $(n(n-3) / 2)+1=((n-1)(n-2) / 2)$ triangularization equations of the form (6.1). These remaining equations are the triangularization equations for the general $S L(n-1, \mathbb{C})$ patching matrix.

Since it was assumed at the beginning that an approximation and triangularization procedure for the $S L(n-1, \mathbb{C})$ patching matrix existed, the remaining triangularization equations can be solved. We have therefore given a construction by which the approximation and triangularization procedure for the $S L(n, \mathbb{C})$ patching matrix is reduced to that of the $S L(n-1, \mathbb{C})$ case, so the inductive argument is complete.

\section{Discussion}

Throughout the above, it was assumed that the patching matrix $P$ is effectively a freely specified holomorphic $S L(n, \mathbb{C})$ function of three variables. By subjecting $P$ to the reality conditions $P(z)^{\dagger}=P\left(z^{*}\right)$, where ${ }^{\dagger}$ represents the adjoint of the matrix and $*$ represents the appropriate complex-conjugation on twistor space, it becomes a freely specified holomorphic $S U(n)$ function of three variables and for $n=2$ the triangularized approximate patching matrix $P_{T}^{(m)}$ becomes equivalent to an element of the Ward Ansätz $\mathscr{A}_{m}$ [5]. Imposing these reality conditions does not affect the convergence or density arguments given for the approximate $S L(n, \mathbb{C})$ patching matrix and so these same arguments confirm the hypothesis of the density of the $\mathscr{A}_{m}$ in the $S U(2)$ solution space. It is not always, however, entirely straightforward to articulate reality and symmetry conditions on upper triangular data, see [5] for some examples. It is worth noting that the set of solutions for the $S L(n, \mathbb{C})$ ASDYM equations with triangular data includes all anti-self-dual instantons and monopoles $[3,5]$.

We have shown that an $S L(n, \mathbb{C})$ patching matrix can, locally, be approximated by a patching matrix which can be triangularized; this triangularized patching 
matrix can be split into two matrix-valued functions from which a local ASDYM connection, associated with this approximate $S L(n, \mathbb{C})$ patching matrix, can be obtained. Since a general $S L(n, \mathbb{C})$ patching matrix can be approximated in this manner, this shows that the set of solutions for the $S L(n, \mathbb{C})$ ASDYM equations for triangular data forms a dense subset (in the Weierstrass sense) in the solution space. Since there exists a method for obtaining explicit solutions in terms of quadratures for triangular data, the ASDYM equations can be thought of as essentially "solved.")

Acknowledgements. We thank George Sparling and John Porter for valuable comments. ETN thanks the NSF for support on grant \#PHY380023.

\section{References}

1. Ward, R. S.: On self-dual Gauge fields, Phys. Lett. 61A, 81 (1977)

2. Yang, C. N.: Condition of self-duality for $S U$ (2) Gauge fields on Euclidean four-dimensional space, Phys. Rev. Lett. 38, 1377 (1977)

3. Atiyah, M. F., Ward, R. S.: Instantons and algebraic geometry. Commun. Math. Phys. 55, 117 (1977)

4. Newman, E. T.: Source-free Yang-Mills theories. Phys. Rev. D18, 2901 (1978)

5. Ward, R. S.: Ansätze for self-dual Yang-Mills fields. Commun. Math. Phys. 80, 563 (1981)

6. Ward, R. S.: Completely solvable Gauge-field equations in dimensions greater than four. Nucl. Phys. B236, 381 (1984)

7. Woodhouse, N. M. J.: Twistor description of the symmetries of Einstein's equations for stationary axisymmetric space-time. Class. Quant. Grav. 4, 799 (1987)

8. Ward, R. S.: Integrable and solvable systems and relations among them. Philos. Trans. R. Soc. Lond. A315, 451 (1985)

9. Woodhouse, N. M. J., Mason, L. J.: The Geroch group and non-Hausdorff twistor spaces. Nonlinearity 1, 73 (1988).

10. Mason, L. J., Sparling, G. A. J.: Nonlinear Schrödinger and Korteweg-de Vries and Reductions of Self-Dual Yang-Mills. Phys. Lett. A 137, 29

11. Corrigan, E. F., Fairlie, D. B., Yates, R. G., Goddard, P.: The construction of self-dual solutions to $S U(2)$ Gauge theory. Commun. Math. Phys. 58, 223 (1978)

12. Newman, E. T.: Gauge theories, the holonomy operator, and the Riemann-Hilbert problem. J. Math. Phys. 27, 2797 (1986)

13. Chakravarty, S., Newman, E. T.: Quadratures for self-dual $G L(2, \mathbb{C})$ Yang-Mills fields. J. Math. Phys. 28, 334 (1987)

14. Mason, L. J., Chakravarty, S., Newman, E. T.: Bäcklund transformations for the anti-self-dual Yang-Mills equations. J. Math. Phys. 29, 1005 (1988)

15. Pressly, A., Segal, G.: Loop groups, Oxford: Oxford University Press 1986

16. Muskhelishvili, N. I.: Singular integral equations. Groningen: Noordhoff 1953, Chap. 5

17. Clancey, K., Gohberg, I.: Factorization of matrix functions and singular integral operators. Basel: Birkhäuser 1981, Chap. IV

18. Woodhouse, N. M. J.: On self-dual gauge fields arising from twistor theory. Phys. Lett. A 94A, 6, 269 (1983)

19. Woodhouse, N. M. J.: Real methods in twistor theory. Class. Quant. Grav. 2, 257 (1985)

20. Hille, E.: Analytic function theory, vol. 1, Blaisdell 1959, p. 219

21. Hille,: p. 209

22. Hille,: p. 70

23. Dettman, J. W.: Mathematical methods in physics and engineering, New York: McGraw Hill 1969, p. 16

24. Hille,: pp. 190-191, 205-207

Communicated by S.-T. Yau

Received October 16, 1989 
